\title{
ATYPICAL PAIN IN ANGINA PECTORIS AND MYOCARDIAL INFARCTION
}

\author{
BY \\ J. D. SPILLANE * AND PAUL WHITE \\ From the Massachusetts General Hospital, Boston, U.S.A. \\ Received November 6, 1939.
}

Pain resulting from disease of the coronary arteries is characterized by its location in the chest, usually behind the sternum, and by its paroxysmal nature. In some instances, however, the pain may be entirely extrathoracic and not necessarily paroxysmal. We wish to draw attention to two groups of patients suffering from coronary sclerosis with such atypical pain. In the first group the pain occurs paroxysmally, is related to effort, but is eccentrically placed; the attack so occasioned resembles classical angina pectoris in all other respects. In the second group are instances of angina pectoris and myocardial infarction in which chronic left shoulder pain is a prominent feature; the latter may herald the complete attacks or follow in their wake.

\section{Group 1. Patients with Eccentrically placed Anginal Pain}

A long-recognized example of these larval or fractional forms of angina pectoris is that in which pain on effort appears only in the arms, the crucial substernal pain being absent. The true nature of such cases was recognized by Heberden (1786). One of his patients suffered from repeated attacks of pain in the left arm, on effort or at night, from 60 until his sudden death at the age of 75 ; he never experienced any chest pain. Since then many others (Mackenzie, 1923, Osler, 1910, and Allbutt, 1915) have described similar cases and commented on the diagnostic puzzle they may present. Mackenzie observed one in whom the pain was confined to the left little finger : and Allbutt one, who felt his pain in the centres of the palms of both hands on walking rapidly or on dancing; some time later, however, he developed the full-dress type of angina and died in an attack. Osler quoted the case of Lord Clarendon, as noted by Blackall in his famous book on the dropsies, in whom the pain was always brachial, even in the fatal attack. Osler described patients where the pain was felt in the middle of both forearms or at the wrists or elbows, whose further course provided indisputable evidence of angina pectoris.

Somewhat similar, but less likely to cause confusion, is the case in which the pain commences distally and spreads rapidly towards the chest (Potain's

\footnotetext{
* Commonwealth Fund Fellow at Harvard Medical School.
} 
angine renversée). Such pain commonly begins in the region of the wrists or elbows and may arrest the patient in his track before it has spread upwards to the chest.

Notes of our 12 cases are given in Table I and short notes of 3 of them follow.

TABLE I

Paroxysmal Arm Pain, on Effort, before Onset of Classical Angina

\begin{tabular}{|c|c|c|c|c|c|c|c|}
\hline \multirow{2}{*}{$\begin{array}{c}\text { Case } \\
\text { No. } \\
1\end{array}$} & \multirow{2}{*}{ Age } & \multicolumn{4}{|c|}{$\begin{array}{c}\text { Site and Duration of Pain before Cardiac } \\
\text { Symptoms appeared }\end{array}$} & $\begin{array}{l}\text { Subsequent } \\
\text { Course }\end{array}$ & \multirow{2}{*}{$\begin{array}{c}\begin{array}{c}\text { Electro- } \\
\text { cardiogram }\end{array} \\
\mathrm{T}_{\mathbf{2}}, \mathrm{T}_{\mathbf{3}} \text { inverted }\end{array}$} \\
\hline & & Both wrists an & d e & & . 1 yr. & $\begin{array}{l}\text { Typical angina, } \\
\text { bilateral radiation }\end{array}$ & \\
\hline 2 & 52 & Both forearms & & $\cdots$ & 2 yr. & Cardiac infarction & $T_{1}, T_{2}$ inverted \\
\hline 3 & 63 & Both upper ar & $\mathrm{ms}$ & . & $6 \mathrm{mo}$ & Cardiac infarction & $\mathrm{T}_{2}, \mathrm{~T}_{3}$ inverted \\
\hline 4 & 50 & Left arm & .. & . & $5 \mathrm{mo}$ & $\begin{array}{l}\text { Typical angina, } \\
\text { no radiation }\end{array}$ & L.A.D. \\
\hline 5 & 48 & Left arm & $\cdots$ & $\cdots$ & . 4 yr. & " $\begin{array}{c}\text { Angine } \\
\text { versée }\end{array}$ & Late inv. $T_{1}$ \\
\hline 6 & 63 & Both wrists & . & . & . 2 mo. ${ }^{*}$ & Not improved & Inv. $T_{2}, T_{3}$ \\
\hline 7 & 61 & Right forearm & & & . 2 yr.* & Not improved & Low $T_{2}$, inv. $T_{3}$ \\
\hline 8 & 60 & Left arm & & & . 8 yr. & Cardiac infarction & - \\
\hline 9 & 56 & Left elbow anc & d fo & $\mathrm{rm}$ & .. $25 \mathrm{yr}$ & Cardiac infarction & Inv. $T_{2}, T_{3}, T_{4}$ \\
\hline 10 & 52 & Both forearms & $\ldots$ & . & . $4 \mathrm{mo}$. & Cardiac infarction & Low $\mathrm{T}_{2}$, inv. $\mathrm{T}_{3}$ \\
\hline 11 & 61 & Left face & . & $\cdots$ & . 3 yr. & $\begin{array}{l}\text { Later involved } \\
\text { L. neck and arm }\end{array}$ & $\underset{\text { diphasic }}{\mathrm{T}_{1} \text { inv., }} \mathrm{T}_{2} \mathrm{~T}_{3}$ \\
\hline 12 & 50 & Left arm & .. & . & .. 8 yr. & Classical angina & \\
\hline
\end{tabular}

* No classical angina.

Case 1. Pain in both wrists, on effort; typical angina 1 year later

Male, aged 56. Acute pain over flexor surfaces of both wrists and elbows, on exertion, for 2 years. No chest pain. No arthritis. Attacks relieved by rest, but sometimes last twenty minutes. More severe in left arm, with sensation as though the sphygmomanometer cuff was applied. Typical substernal angina of effort, with radiation to left arm, for one year ; relieved by nitrites. Distant heart sounds ; no cardiac enlargement. B.P. 130/80. EC., $T_{2}$ and $T_{3}$ inverted; prominent $Q_{3}$.

Case 5. Pain in left arm on exertion; " angine renversée" 4 years later.

An obese woman, aged 48, known to have had a blood pressure of $220 \mathrm{~mm}$. at 38 . Four years ago attacks of pain and "deadness" in the entire left arm, lasting a few minutes and provoked by hurry and bustle. No other symptoms till one night, after hurrying to prepare supper, she was seized with an agonizing pain in left arm, which incapacitated her. A dozen similar attacks followed in the next 24 hours and culminated in a paroxysm in which pain started in left wrist and spread slowly up to left shoulder and for the first time to the chest and substernal region. Morphia was required. The left chest was held as if in a vice. Subsequently developed classical angina, with radiation to left arm and relief with nitrites. B.P. 185/95. EC., late inversion of $T_{1}$.

Case 8. Pain in left upper arm on exertion; classical angina 8 years later

Male, aged 60. Sharp pain in left arm above elbow and sometimes in forearm on walking briskly or on stairs for 8 years, usually with dyspnœa. No other symptoms. No physical signs and X-ray of heart and EC. normal. Two years later attacks recurred, with more dyspnœa. After another 2 years they became more frequent and severe ; 
angina pectoris was suspected and nitroglycerine was at once successful. Attacks continued during the next 4 years but no substernal pain till 3 months before observation, when it started in the chest and spread down left arm. He died suddenly in hospital and autopsy confirmed an anterior myocardial infarct.

\section{Discussion}

No satisfactory explanation for the referred pains of angina pectoris is forthcoming to account for all the clinical features and for some of the unexpected results of sympathectomy. According to the theory of Head and Mackenzie a stimulus passes from the heart via the sympathetic to the posterior root ganglia, where it is transformed and leads to stimulation of certain spinal ganglion cells with the appreciation of pain. This pain is felt along the distribution of the nerve root in question and so it is called referred pain. This explains the occurrence of extrathoracic pain of cardiac origin, but does not account for the inverse radiation of the pain in other instances, where it seems as if the pain originates in the periphery and passes towards the centre. The hyperæsthesia sometimes found with such seizures would suggest this, just as any severe peripheral pain may leave hyperæsthesia of the skin. Such patients often liken their arm pain to that produced by prolonged inflation of a sphygmomanometer cuff. Robertson and Katz (1938) maintained the sphygmomanometer cuff in position for five minutes, at a pressure $50 \mathrm{~mm}$. above the systolic pressure, and found that the subsequent arm pain was followed by anginal paroxysms in 19 out of 24 patients who were subject to angina and were tested in this way. When repeated on the right arm, anginal attacks only followed if the pain radiated to that arm in the spontaneous attacks. When applied to the legs the tests failed. These results suggest that by producing one element of the spontaneous attack, viz. the pain in the arm, the fully developed paroxysm may be induced. By what mechanism it is brought about we do not know. On repeating these experiments we have found that, although we were unable to reproduce anginal attacks in more than half of our anginal patients, the "squeezing" or "gripping" sensations in the arm, in both the induced and spontaneous attacks, were indistinguishable. It is possible they are produced by the same mechanism, viz. ischæmia of the forearm muscles. Does spasm of smooth muscle occur in the left arm during anginal seizures ?

Penfield (1925) has shown that removal of a sympathetic ganglion takes away the possibility of angina, only in the motor distribution of that ganglion : it is still possible in the motor distribution of the remaining ganglia. He pointed out that the success of such an operation depends, not upon the interruption of a direct afferent path from cardiac sympathetic plexus to central nervous system, but upon the interruption of autonomic reflexes. Accordingly, he suggested that the nervous mechanism in angina pectoris depends on an autonomic reflex. It causes peripheral spasm of smooth muscle and may resemble the pain of various angiospastic conditions. This theory differs from Head's in that the point of contact between sympathetic and cerebrospinal systems is shifted from the posterior root ganglia to the periphery. That such an explanation is not unlikely is suggested by the replacement of pain in some 
anginal attacks by paroxysms of homolateral sympathetic stimulation (Gibson, 1905 ; Palmer, 1930 ; Holt, 1930).

The case histories outlined above emphasize the importance of studying closely any middle-aged or elderly person complaining of bouts of pain in the arm on exertion. Many such attacks, sooner or later, blossom into typical angina, but in others sudden death may occur before the coronary nature of the attacks is suspected.

\section{Group II. Persistent Pain in the Shoulder}

A symptom that is less readily identified as due to disease of the coronary arteries is chronic pain in the shoulder and arm, not affected by exercise and not relieved by nitrites. The pain does not occur in crises, but is more persistent, lasting for hours at a time and very intractable to ordinary therapeutic measures. Howard (1930) published reports of five patients with a stiff painful shoulder in association with coronary sclerosis, syphilitic aortitis, or cardiac infarct. Lian (1931) has described similar cases and concluded that a " thoracobrachial neuralgia" resulted from the repeated shocks to the brachial plexus by the anginal paroxysms. He mentioned the similarity to arthritis of the shoulder. Edeiken and Wolferth (1936) reported 14 cases with persistent pain in the shoulder following myocardial infarction, and Boas and Levy (1937) described 21 cases. In 29 of these 40 cases the left shoulder was involved, but some patients with radiation of the anginal pain to the right shoulder subsequently developed chronic right shoulder disability.

TABLE II

Chronic Shoulder Pain preceding Angina Pectoris or Cardiac Infarction

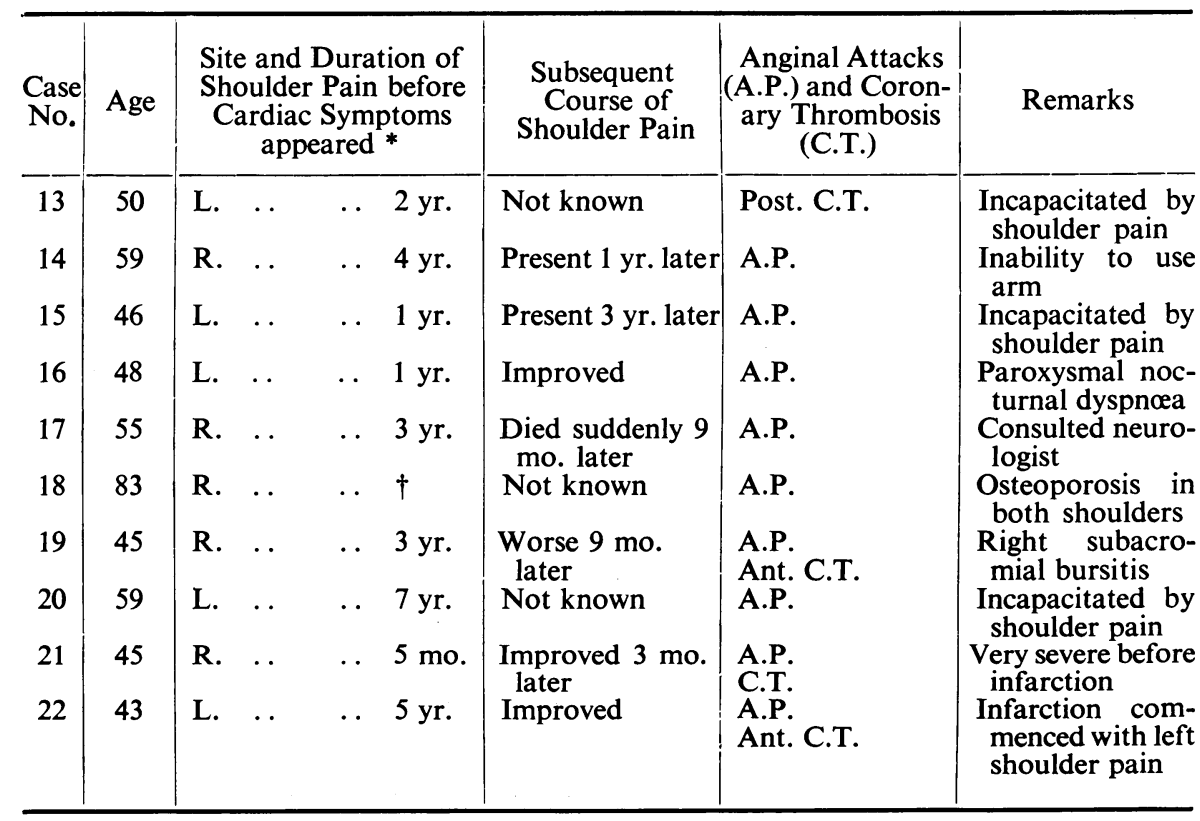

* L. and R. indicate shoulder affected.

$\uparrow$ Simultaneous onset 1 year before. 
In the present series are 25 patients with angina pectoris and/or myocardial infarction, in whom chronic shoulder and arm pain formed a prominent complaint. In 10 the shoulder complaint preceded the angina or infarction by months or years (Table II). In 15 it followed and was the outstanding feature during convalescence (Table III).

TABLE III

Chronic Shoulder Pain following Angina Pectoris or Cardiac Infarction

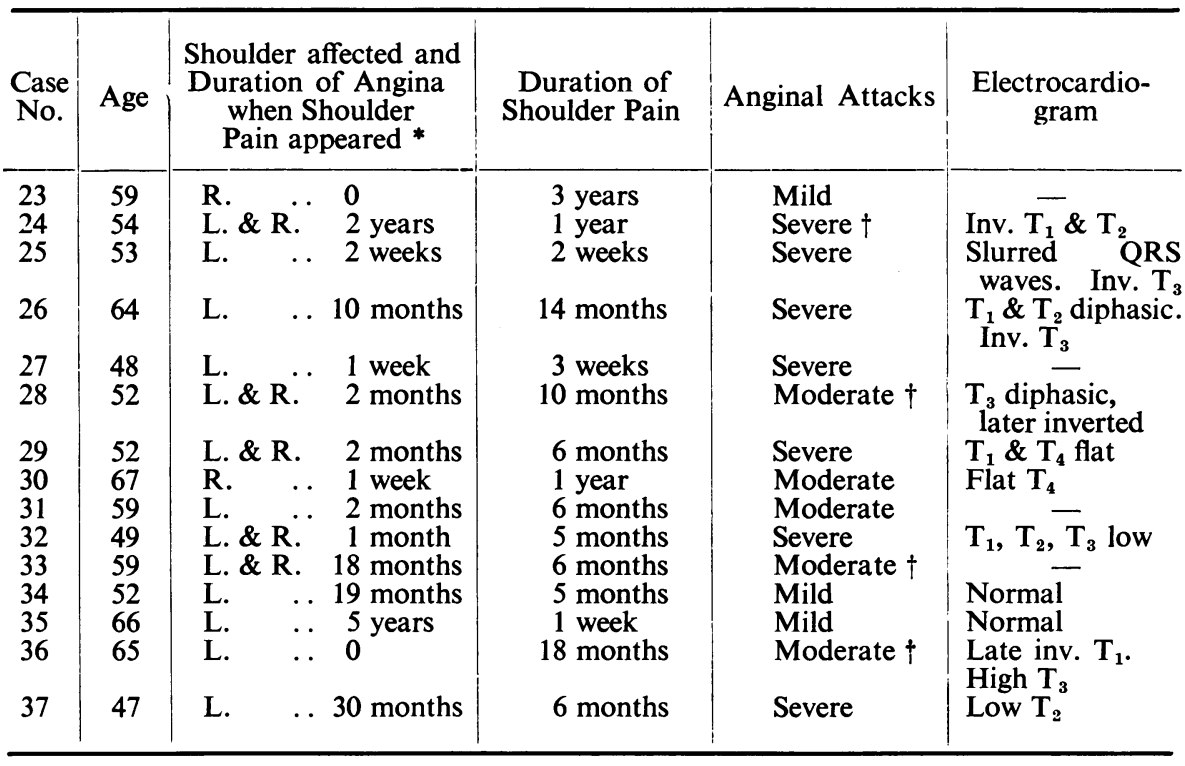

* L. and R. indicate the shoulder affected.

$\dagger$ A diagnosis of myocardial infarction was made in these cases.

Case 13. Chronic pain in left shoulder for 2 years before cardiac infarction

Male, aged 50. Dyspepsia for 15 years. Dyspnœa on exertion and pain, not related to exertion and lasting several hours, twice a week, in left shoulder and arm, for past 2 years. No chest discomfort. Some general weakness and pallor for 6 months. Chronic shoulder pain had increased in severity lately, preventing sleep. Forced to give up work because of it 6 months ago. Weight loss of $15 \mathrm{lb}$. during past year. Two weeks ago pain became much worse and he entered hospital. Dyspnœic. B.P. 150/85. EC. showed inverted $T_{2}$ and $T_{3}$, and prominent $Q_{3}$. Gradual improvement while under observation.

Case 22. Recurrent persistent pain in left shoulder for 5 years before cardiac infarction

Male, aged 43. Well and active till 5 years ago. Then pain in left shoulder and down the arm to fingers, persisting, on and off, for 2 years; artificial heat and light prescribed without improvement. A few months ago at night, a severe grinding pain in the left elbow and forearm spreading upwards to the shoulder, and lasting an hour. Next day the left arm pain was worse and he felt exceedingly ill, with slight fever, and was in bed for several weeks. Now complains of typical angina, spreading to left arm. B.P. 150/90. EC. showed lead III inverted. 
Case 24. Persistent pain in both shoulders for 1 year; angina for 3 years

Male, aged 54. Angina pectoris 3 years. No radiation. Attacks becoming more frequent, till three times a day ; incapacitated for 2 years. One year persistent pains in both shoulders, especially left, radiating down both arms in anginal attacks. Two severe attacks 6 weeks ago in which pain was persistent and unaffected by nitrites ; " terrific" pain in left shoulder during one attack, since when pain in left shoulder and arm almost constant. Aortic regurgitation. B.P. 230/60. Heart enlarged to left. EC., inverted $\mathrm{T}_{1}$ and $\mathrm{T}_{2}$.

\section{Case 25. Persistent left shoulder pain 2 weeks after onset of angina}

Male, aged 53. Anginal attacks for one month with radiation to left arm. Three severe attacks in all before onset of constant pain in left shoulder; this had been present 2 weeks when examined. Some dyspnœa on exertion. B.P. 155/80. Moderate enlargement of heart. Aorta slightly tortuous. EC., $\mathrm{T}_{3}$ inverted. X-ray of left shoulder, no abnormality. Pronounced atrophy of left shoulder-girdle muscles, with moderate limitation of all voluntary but not of passive movements. No tenderness or fullness in region of subacromial bursa.

\section{Discussion}

In this group we have described an affection of the shoulder and arm in patients with angina, characterized by constant dull pain, limitation of movement, and weakness or lameness of the arm. The pain is not usually provoked by exercise, but may be aggravated by use of the affected arm, as for carrying. Examination of the shoulder reveals no local lesion. Calcification in the subdeltoid bursa or of the supraspinatus tendon is rarely encountered. The pain is intractable, not unlike a causalgia, and may shift from one shoulder to the other. Usually, in angina with left-sided radiation, it affects the left shoulder and arm ; with right-sided radiation, the right shoulder and arm. This is by no means constant, however, and in this sense there was agreement in 6 cases and disagreement in 2, the remainder showing no radiation. Tenderness over the shoulder is infrequent, but atrophy of the shoulder girdle muscles has been seen. In Case 25 pronounced atrophy of the muscles of the left shoulder girdle developed four weeks after a myocardial infarction. Active abduction and external rotation were limited but passive movements were normal. No tenderness, crepitus, or pain on manipulation were discovered ; $\mathrm{X}$-ray showed no abnormality. Many patients complain that the pain is much worse at night and prevents sleep.

The disability may precede or follow angina pectoris or cardiac infarction ; if present before infarction occurred it is usually intensified. The shoulder pain may also be the outstanding feature of the coronary occlusion, overshadowing the substernal pain. In those instances where shoulder pain preceded the appearance of other symptoms, relief was frequently sought of the neurologist, orthopædist, or physiotherapeutist. Sedatives, massage, and heat are usually without much success, and morphia is often needed in the acute phase. The administration of nitrites has no effect upon the constant aching, unlike the success frequently encountered in the eccentric paroxysmal pain of the first group. It thus seems that vascular spasm plays no part in 
its production. The affection appears to run its course despite treatment and subsides spontaneously.

The mechanism of this shoulder and arm pain is obscure. There is rarely any local lesion to account for it. A few authors, however, have described the intensification of the symptoms of subacromial bursitis following a myocardial infarction (Libman, 1935 and Boas and Levy, 1937), and one such case is to be found in our series (Case 19). It is doubtful if this is the explanation for all such cases of shoulder pain. In the absence of localized tenderness or fullness beneath the deltoid or calcification within the sac, it would be difficult to make a diagnosis of bursitis. Libman thinks that one frequently finds spondylitis, bursitis, intercostal neuralgia, and other "gouty" disorders associated with true angina pectoris, because they have the same origin.

But in our patients there was no evidence of a previous bursitis, and even during the height of the disability no indication of a local lesion. The pain was not always felt in the shoulder region, but sometimes in the muscles of the arm or forearm when movements at the shoulder were entirely free. At times the pain was replaced by a "deadness" or "heaviness" or "lameness." The hand of the affected arm was sometimes cold and numb ; cyanosis, pallor, or œdema of the arm were not observed. Similar transient symptoms and signs are wellknown sequels to attacks of angina pectoris. In our patients these symptoms are intensified and prolonged ; they are the main complaint, differing only in degree and duration from the transient post-anginal phenomena. A localized lesion in the shoulder has not been invoked to explain the latter and is probably unnecessary in the former.

Much of the discussion on the extra-cardiac site and radiation of cardiac pain dates from the observation by Mackenzie (1923) that in one of his patients with an abscessed tooth the anginal pain radiated to that tooth; yet irrefutable instances are rarely, if ever, encountered in actual practice. Even if a local lesion is demonstrated in the path of an eccentrically radiating anginal pain, the obvious conclusion is highly dangerous, as twice as many instances of similar radiation can be cited in which no obvious local lesion existed. Such was the condition in Case 2, where the facial localization of the pain led to a suspicion of trigeminal neuralgia.

In view of the hypotheses put forward to account for the bizarre radiation of some anginal pains and in view of the popularity of the concept of " sensitization of pathways," it is instructive to consider the following history. A man, aged 60, had sustained a compound fracture of the right arm 14 years previously, necessitating amputation below the shoulder. The pain in the stump became constant and intolerable, so that on several occasions novocain injection was performed. In the absence of any relief, and because of the severity of the pain, numerous surgical procedures were attempted during the following 7 years-section of roots of the brachial plexus, repeated novocain blocks, laminectomy with section of roots, and alcoholic injection of the stellate ganglion-but the pain still continued, when anginal attacks made their appearance. The pain radiated to the left arm, and was relieved by nitrites. A more persistent pain in the left arm was unrelieved by nitrites and unaffected by 
exercise. Novocain injection of the left stellate ganglion afforded much relief. The patient died suddenly of myocardial infarction. Such an example lends little credence to the theory of "sensitization of pathways" in the determination of the radiation of anginal pain. The right brachial plexus had been subjected to repeated surgical procedures and causalgic pains were intractable for 14 years, yet subsequent anginal pains radiated to the left arm.

To return to our main thesis. What, then, is the nature of persistent shoulder and arm pain in coronary arterial disease? In some there may be a local lesion of muscle or bursa aggravated by the cardiac nerve irritability, but in others there may be simply an irritation of the cardiac sympathetic plexus in the presence of myocardial ischæmia. In favour of the former theory, shoulder pain is extremely common whether there is angina pectoris or not, but such an explanation seems unlikely for every case. The pain is referred to the shoulder and arm, usually of the left side, and is associated with the attacks of angina pectoris. The constant nature of the pain could be accounted for on the basis of the theory of " summation." Intermittent showers of impulses, travelling over pain fibres, may induce constant pain through summation at synapses ; the central neurones, then, most probably discharge continually and give rise to constant pain.

\section{CONCLUSIONS}

Disease of the coronary arteries may manifest itself by paroxysmal, extrathoracic pain on effort. Twelve cases are described in which bouts of pain in one or both arms were, for varying periods of time, the only manifestation of ill health. Such pains, constricting in character, appear suddenly on exertion ; they are felt usually at the wrists, forearms, or elbows, and are relieved by rest or by nitroglycerine. Fatal attacks may ensue, attacks in which the pain remains located in the arm. In others typical anginal paroxysms or cardiac infarction subsequently develop.

Classical angina pectoris or cardiac infarction is frequently complicated by persistent pain in the shoulder and upper arm. This pain is of a dull, aching character, diffusely felt, usually on the left side in left-sided and on the right side in right-sided anginal attacks. The pain bears no direct relation to exercise and is not relieved by nitroglycerine. It may precede or follow angina pectoris or cardiac infarction by weeks or even by years. Ten cases are described in which chronic shoulder and arm pain was followed, 5 months to 5 years later, by typical angina pectoris. This pain may be incapacitatingly severe during accesses of coronary insufficiency and may then be the outstanding therapeutic problem. Fifteen cases of similar pain following angina pectoris or cardiac infarction are recorded.

The mechanism of the chronic pain is obscure. A local lesion of the shoulder or arm can rarely be demonstrated, though exacerbation of a pre-existing subacromial bursitis may be the explanation in some cases. Often the pain may be interpreted as one of the varied referred phenomena encountered in the arms in angina pectoris and cardiac infarction. 


\section{REFERENCES}

Allbutt, C. (1915). Diseases of the Arteries including Angina Pectoris. London. Vol. 2, p. 299.

Boas, E. P., and Levy, H. (1937). Amer. Heart J., 14, 540.

Edeiken, J., and Wolferth, C. C. (1936). Amer. J. med. Sci., 191, 201.

Gibson, A. (1905). Brain, 28, 197.

Heberden, H. (1786). Med. Trans. (College of Physicians). London, 2, 59.

Holt, E. (1930). Amer. Heart J., 5, 522.

Howard, T. (1930). Med. J. and Rec., 131, 364.

Lian, C. (1931). J. de méd. et chir. prat., 102, 153; and Rev. belge Sci. méd., 3, 354.

Libman, E. (1935). Bull. N. Y. Acad. Med., 11, 427.

Mackenzie, J. (1923). Angina Pectoris. Hodder and Stoughton, London.

Osler, W. (1910). Lancet, 1, 697.

Palmer, R. S. (1930). Amer. Heart J., 5, 519.

Penfield, W. (1925). Amer. J. med. Sci., 170, 864.

Robertson, S., and Katz, L. N. (1938). Ibid., 196, 199.

Spillane, J., and White, P. D. (1939). Brit. Heart J., 1, 291. 